\title{
sur la gestion et les finances publiques Spécial COVID-19 en Juin 2020
}

\section{BUDGET DE L'ÉTAT ET DES OPÉRATEURS}

\section{$\pi$ Politique budgétaire}

\section{Une synthèse des mesures d'urgence}

Le rapport de la mission d'information de l'Assemblée nationale, nº 3053 du 3 juin, sur l'impact, la gestion et les conséquences de l'épidémie présenté par Richard Ferrand contient un résumé précis des mesures économiques et sociales pour faire face à la crise : -Mesures de soutien aux entreprises : fonds de solidarité pour les très petites entreprises (3,4 Md€ au 27 mai), prêts garantis par l'État (400 000 dossiers représentant 66,46 Md€ d'encours de prêts plus des prêts du FDES et des avances remboursables de trésorerie), report de charges fiscales et sociales (estimé à 42 MdE dans le PLF2 et accélération du remboursement des crédits d'impôts et de TVA), aides spécifiques à certaines catégories d'entreprises (export, startups, entreprises stratégiques, aides sectorielles pour le tourisme, la construction, la culture, le transport aérien) - Mesures de soutien à l'emploi et aux salariés : extension du dispositif d'activité partielle, exonération des heures supplémentaires, assouplissement de la prime de pouvoir d'achat, primes pour les soignants et les autres fonctionnaires mobilisés - Mesures de soutien aux ménages les plus modestes : allocation exceptionnelle de solidarité pour les bénéficiaires de minima sociaux, aide exceptionnelle en faveur des jeunes de moins de 25 ans.

\section{$\lambda$ Textes budgétaires}

Troisième PLFR : I'État au secours de l'économie

Le projet de loi de finances rectificative (PLFR), présenté le 10 juin en conseil des ministres, table sur une récession de l'économie française de $11 \%$ du PIB en 2020, prévision que le Haut conseil des finances publiques estime "prudente » dans un avis publié le 8 juin. Le document note également que la prévision du déficit s'établit à 222,1 Md€, soit 11,4 points de PIB (-2,2 \% en LFI 2020), un niveau jamais atteint depuis la fin de la Seconde Guerre mondiale. Le ratio des dépenses publiques s'établit à 63,6\% du PIB et celui de la dette à $121 \%$. Le nouveau PLFR intègre notamment le financement de plusieurs plans sectoriels de soutien : $18 \mathrm{Md}$ € pour le tourisme, $10 \mathrm{Md}$ pour l'automobile, $15 \mathrm{Md} €$ pour l'aéronautique, $700 \mathrm{ME}$ en faveur des startups et entreprises technologiques, 1,3 Md€ pour le secteur culturel. II prévoit aussi des mesures de soutien pour les collectivités territoriales et d'exonérations sociales pour les PME.

\section{$\pi$ Dette publique}

\section{Le cantonnement de la dette publique}

Le 10 juin, la Banque de France a réalisé une courte note sur les leçons historiques sur la dette publique. Elle constate que, même si au $X X^{e}$ siècle de fortes augmentations de la dette ont suivi les deux guerres mondiales ainsi qu'une forte inflation, il n'en a pas forcément été de même pour les guerres napoléoniennes ou du XVIII siècle, la solution du cantonnement ayant permis de reporter les perspectives de remboursement sans limiter les marges de manœuvre budgétaire à court terme. Le 29 juin, à l'Assemblée nationale, le ministre de l'économie et des finances, Bruno Lemaire, envisage le cantonnement de $150 \mathrm{Md} €$ de dette liée à l'épidémie et leur amortissement par la CADES ou une autre caisse. II serait financé par l'affectation d'une part de TVA ou une CRDS prolongée jusqu'en 2042. L'Allemagne envisage 
un dispositif de cantonnement de $118 \mathrm{Md}$ de dette COVID sur la période 2023-2042.

\section{FINANCES LOCALES}

\section{$\pi$ Données générales sur les finances lo- cales}

\section{L'impact de la crise}

Le 10 juin, lors d'une conférence de presse, Jean-René Cazeneuve a livré un certain nombre d'enseignements sur le contenu à venir de son rapport ( $v$.Repères de mai). Ainsi, les pertes fiscales et tarifaires des collectivités territoriales s'élèveraient à 7,5 Md€ en 2020, soit 3 à $4 \%$ de leurs recettes réelles de fonctionnement. Les DMTO accuseraient cette année une baisse de $25 \%$ et le versement mobilité de $20 \%$. Lors de cette conférence de presse, J.-R. Cazeneuve a également indiqué que le fonds d'aide prévu par la troisième loi de finances pour 2020 couvrirait environ $88 \%$ des recettes des structures concernées avec cependant quelques collectivités insuffisamment aidées. Le député a également émis l'hypothèse d'aborder la question des impôts de production. Enfin, s'appuyant sur les dernières hypothèses économiques favorables pour 2021, il n'exclut pas la piste d'un rebond important des recettes fiscales dès 2021.

\section{Le plan de 4,5 Md€ dans le PLFR3}

La troisième loi de finances rectificative pour 2020 déposée le 10 juin 2020 ( $n^{\circ}$ 3074) continue d'ajuster les finances locales avec la crise sanitaire. Ainsi, ce projet de loi prévoit d'aider les communes et les établissements publics de coopération intercommunale (EPCl) en difficulté à hauteur de 750 millions des pertes de recettes fiscales et domaniales. Pour les départements, près de 2,7 Md€ d'avances seront déployées pour les collectivités qui connaissent des pertes de recettes de droit de mutation à titre onéreux (DMTO). Des dispositifs sont également prévus pour aider les collectivités ultra-marines. Le projet de loi de finances prévoit la possibilité pour les communes et EPCl d'exonérer de taxes de séjour les hébergeurs.

\section{FINANCES SOCIALES}

\section{$\pi$ Situation des comptes sociaux}

Le débat sur l'apurement de la dette générée par la crise du COVID 19

Dans un courrier de fin mai dont le contenu a été rapporté par Les Echos, le Président de la Fédération hospitalière de France a proposé de reporter jusque vers 2050-2055 I'apurement de la dette sociale logée dans la CADES, que le gouvernement envisage de prolonger jusqu'en 2033 (cf. Repères de mai). II propose de transférer le surcroît de dette sociale consécutif à la crise COVID à l'État ou à une structure de défaisance, commune à l'ensemble des dettes générées par les baisses d'activité générées par le confinement. Cette idée semble avoir fait son chemin au gouvernement (v. supra Budget de l'État)

\section{PLFR 3 pour 2020 : envolée des dépenses sociales}

Le troisième projet de loi de finances rectificative pour 2020 adopté par le conseil des ministres du 10 juin a porté à $134 \mathrm{MdE} \mathrm{le} \mathrm{coût}$ de l'impact de la crise pour le budget de l'État (v. supra budget de l'État). Pour ce qui concerne le secteur social, les principaux surcroîts de dépenses concernent le chômage partiel, pour lequel la prévision d'utilisation passe de $24 \mathrm{Md}$ à $31 \mathrm{Md}$, le Fonds de soutien aux indépendants (porté à $8 \mathrm{Md}$ ) et la charge résultant de l'annulation définitive de $3 \mathrm{Md}$ de cotisations sociales reportées.

\section{Commission des Comptes de la Sécurité sociale : le déficit 2020 pourrait atteindre $52 \mathrm{Md} €$}

Le rapport de la Commission des comptes de la SS, publié le 16 juin, rend compte des exercices 2019 et 2020. S'agissant des comptes 2019, le déficit cumulé du Régime général et du FSV s'établit finalement à -1,9 Md€, soit beaucoup moins que le montant prévu par la LFSS de 2020 $(-5,4 \mathrm{Md})$. Le RG est quasiment à l'équilibre $(-0,4 \mathrm{Md})$-avec deux branches, maladie et vieillesse, en négatif d'environ -1,5 Md€, le déficit du FSV est ramené à $-1,5 \mathrm{Md}$. Ce résultat est dû à un surcroît de recettes de cotisations et de CSG de 2,4 MdE, dû principalement à la dynamique du revenu des indépendants, à la croissance des prélèvements sur les revenus du capital et à une moindre progression des dépenses de retraites (minimum contributif) ainsi qu'à un strict respect de I'ONDAM. Sur la base des hypothèses économiques associées au $3^{e}$ PLFR (baisse du PIB 2020 en volume de -11\%), le déficit du RG et du FSV plongerait de -5,4 Md€ à -52 Md€ (le précédent record de l'année 2010 n'étant « que » de $28 \mathrm{Md}$ ) : maladie -31,1 Md€, accidents du travail -0,7 Md€, retraite -14,9 Md€, famille -3,1 Md€, FSV-2,1 Md€). La plus grande partie de l'écart sera causé par un effondrement des recettes de -42,8 Md€ du fait d'une contraction de la masse salariale de -9,7 \% (-33 Md€ de pertes de cotisations), des difficultés de recouvrement et par une réduction de 9,8 Md€ 
des impôts et taxes affectées, le solde étant constitué par un dépassement de I'ONDAM de $8 \mathrm{Md} €$ du fait des dépenses de santé décidées en LFR $n^{\circ}$ 2. Le rapport ne donne aucun éclairage sur l'année 2021 et au-delà, mais met l'accent sur la situation de l'ACOSS dont le plafond d'emprunt a été réévalué à $95 \mathrm{Md€}$ par le décret du 20 mai 2020 ; la structure des financements de l'ACOSS s'est trouvée totalement bouleversée : au 6 juin, les émissions sur les marchés financiers s'élevaient au montant inédit de $46 \mathrm{Md} €$ et les financements bancaires, qui étaient devenus marginaux ces dernières années, sont remontés à près de $40 \mathrm{Md} €$, dont la moitié apportés par la Caisse des Dépôts.

\section{Santé/Assurance maladie/Hôpital}

6,3 Md€ pour la revalorisation des traitements des personnels hospitaliers

L'un des piliers du "Ségur de la santé " concerne la situation des personnels hospitaliers. Les revendications immédiates paraissent converger vers une augmentation mensuelle de $300 €$ des salaires des infirmiers, aides-soignants et agents des services hospitaliers, ce qui équivaut à une majoration de 85 points de la grille de la fonction publique hospitalière. La perspective ouverte par le gouvernement était de rattraper la moyenne européenne et la dernière modification de la grille salariale hospitalière date de 1988. Le 25 juin, le ministre des solidarités et de la santé a annoncé, lors des réunions de négociation, une enveloppe de $6 \mathrm{Md} €$; mais des incertitudes demeurent sur la forme des revalorisations (hausse de salaire nette, révision des grilles ou primes) et sur le périmètre (qui inclurait les personnels des établissements privés, lucratifs ou non). Peu de temps après, Olivier Véran a ajouté une enveloppe de 300 M€ pour les médecins hospitaliers. L'objectif du gouvernement est de boucler les « accords de Ségur » pour la mi-juillet, mais il se heurte à une fronde croissante des personnels, qui estiment les propositions très en deçà des efforts qu'ont consentis les personnels pendant la crise.

\section{Les complémentaires priées de payer pour les hôpitaux et les soignants}

Les ministres de la santé et des comptes publics ont donné jusqu'à mi-juin aux assureurs complémentaires santé pour présenter des propositions en vue de prendre leur part à l'effort exceptionnel consenti par les professionnels et établissements de santé. Ils se fondent sur les économies de remboursement réalisées par ces organismes du fait de la moindre fréquentation médicale - hors COVID 19 - réalisée pendant la période de l'épidémie et d'une baisse de la sinistralité ordinaire. Les mutuelles et les assurances ne ferment pas la porte à une contribution, mais craignent par-dessus tout l'institution d'une taxe de solidarité à l'occasion des débats parlementaires à venir.

\section{$\pi$ Retraites}

Après un déficit de 30 Mds€ en 2020, la réforme des retraites relancée?

Le Conseil d'orientation des retraites s'est livré le 11 juin à une première analyse des conséquences de la crise du COVID 19 sur les régimes de retraite. II anticipe une faible diminution de la dépense au titre des pensions $(-500 \mathrm{M} €)$, mais une forte augmentation de leur poids par rapport au PIB du fait de la réduction de celui-ci (de 13,7\% à 15,5\%). Du fait de la baisse de l'activité, les ressources des régimes diminueraient fortement $(-25,7 \%)$ et le déficit 2020 passerait de -4,2 Md€ à -25,2 Md€, avec un relèvement du niveau de vie des retraités de l'ordre de $5 \%$ par rapport à celui du reste de la population. Le document du COR étudie les différents moyens envisagés par les gouvernements pour faire participer les retraités au financement de la crise (désindexation...). Parallèlement, le gouvernement laissait entendre que la réforme des retraites pourrait être remise en chantier pour être adoptée avant 2020, avec l'objectif de préserver les volets les plus emblématiques de la réforme systémique, à savoir le passage au régime par points et la suppression des régimes spéciaux.

\section{त Emploi/Formation professionnelle}

\section{Plan de sauvetage de l'apprentissage et pour l'emploie des jeunes}

La crise sanitaire fragilise l'apprentissage qui, après sa progression spectaculaire de l'année 2019, risque de voir le nombre des embauches s'effondrer à la rentrée 2020. Le gouvernement annonce le 2 juin une réflexion sur plusieurs pistes: prime à l'embauche quelque soit le niveau de diplôme (actuellement limité au bac et en deçà), prise en charge des salaires de la première année, extension à six ou douze mois de manière pérenne de la période durant laquelle un jeune peut rester en CFA sans avoir trouvé un emploi, les opérateurs de l'apprentissage souhaitant se voir garantir le même niveau de crédits qu'en 2019. Ces mesures en faveur de l'apprentissage s'inscriront dans un plan massif pour le soutien de l'emploi des jeunes que le 
gouvernement annoncerait pour le début juil-

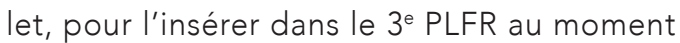
de sa discussion au Sénat. L'option privilégiée serait une exonération pérenne des charges sociales dans une limite d'âge (30 ans) et de salaire (2,5 SMIC), dont le coût (au moins $10 \mathrm{Md}$ ) serait fonction de la population retenue (limitation aux nouveaux contrats ou extension à tous les contrats en cours).

\section{Plan d'aide à l'insertion par l'activité éco-} nomique

Fragilisées par la crise, les 3800 entreprises de I'insertion par l'activité économique (IAE) qui emploient environ 250000 personnes par an pourraient perdre plus du quart de leur chiffre d'affaires en 2020. Elles demandent le dégel des aides aux postes inscrites dans le budget 2020 qui n'auraient pas été dépensées, ainsi que la pérennisation de l'enveloppe d'une centaine de millions d'euros prévue pour le plan d'investissement des IAE.

\section{$\lambda$ Assurance chômage}

Vers un déficit historique de l'Assurance chômage en 2020

Les prévisions présentées au bureau de I'UNEDIC du 18 juin, sur la base des dernières hypothèses économiques associées au PLFR 3, tablent sur la destruction de 900000 emplois à fin 2020 et un taux de chômage de 11 \%. Dans ces conditions, à cette date, le déficit de l'Assurance chômage serait de -25,7 Md€, dont -24,8Md€ imputables à la crise du COVID, parmi lesquels $52 \%$ consécutifs à la prise en charge de l'activité partielle, $29 \%$ à l'augmentation des allocations versées et $19 \%$ aux reports de cotisations. La dette atteindrait 63,1 Md€ fin 2020, contre 37 Md€ fin 2019.

\section{Chômage partiel :évolution en douceur du dispositif}

Alors qu'on s'attendait à un sérieux tour de vis sur les conditions de prise en charge par l'État de l'activité partielle, qui avaient été revues à la baisse au 1er juin, le Président de la République recevant les partenaires sociaux le 24 juin a annoncé une évolution très progressive du dispositif. Le premier volet porte sur l'aménagement du dispositif en place depuis la mi-mars : à compter du 1er octobre 2020, un salarié placé en chômage partiel ne percevra plus que 60 \% de son salaire brut (soit $72 \%$ du salaire net) toujours dans la limite de 4,5 fois le SMIC. L'entreprise sera compensée à hauteur de $60 \%$ (contre $85 \%$ actuellement) ; ce volet vise à traiter les situations de court terme et est destiné aux secteurs dont on peut espérer une sortie de crise rapide. Le second volet repose sur un nouveau dispositif $d^{\prime}$ " activité partielle de longue durée » (APLD) destiné aux secteurs très fortement impactés par la crise (aéronautique, automobile...) : mis en place à partir du $1^{\text {er }}$ juillet, il garantira le maintien de $84 \%$ du salaire net (100\% au niveau du SMIC) et une prise en charge de $85 \%$ pour l'entreprise, qui baissera à $80 \%$ à partir du $1^{\text {er }}$ octobre. Toutefois, I'application du dispositif devra se faire dans le cadre d'un accord d'entreprise ou de branche d'une durée de 6 mois à 2 ans, de façon à préserver l'emploi au maximum (sans pour autant interdire les licenciements) et le volume d'heures non travaillées ne devra pas aller au-delà de $60 \%$. Une clause de revoyure est prévue pour I'automne. Le nouveau dispositif a paru satisfaire à la fois les syndicats et le patronat. Ce mécanisme est extrêmement coûteux pour les finances publiques, et il n'est pas certain que la

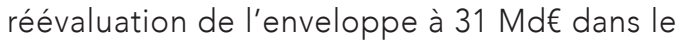
cadre du 3e PLFR (cf. supra) soit suffisante.

\section{$\pi$ Dépendance}

\section{Vers une accélération de la mise en place du risque dépendance?}

En ce qui concerne la création du cinquième risque "dépendance », le projet de loi du gouvernement prévoit la remise pour le $30 \mathrm{sep}$ tembre d'un rapport sur « la création d'un nouveau risque ou une nouvelle branche de la Sécurité sociale » et met en place un financement d'amorçage avec la préemption de 2,3 Md€ de CSG affectée à la CADES à partir de 2024. Les parlementaires de la majorité poussent le gouvernement à se prononcer sur la création d'une $5^{e}$ branche -et non d'un $5^{e}$ risque-, et d'affecter, dès 2021, 0,05 points de CSG affectés à la CADES. Pour autant, il ne semble pas qu'un arbitrage financier sur ce point intervienne avant le PLFSS 2021 qui comportera d'autres sujets particulièrement lourds. Cela au moment où un rapport de I'OCDE pointe le fort retard de la France en matière d'encadrement des personnes âgées dépendantes.

\section{Management public}

\section{$\lambda$ Administration numérique}

\section{L'application Stop-Covid en service}

Après une difficile mise au point, des controverses et l'approbation des plus hautes autorités (cf. Repères covid-19 d'avril et mai), I'appli- 
cation Stopcovid, téléchargeable sur la plupart des téléphones portables, est entrée en service le 2 juin. Elle a pour objet de prévenir les utilisateurs des contacts éventuels avec des personnes testées positives au virus. Elle aurait été téléchargée par 1,9 million de personnes à la mi-juin et aurait détecté 14 personnes à risque. Elle n'est pas interopérable avec les systèmes des pays voisins qui sont, eux, conformes aux prescriptions $d$ e l'Union européenne et basés sur un système de stockage des données décentralisés, qui a l'inconvénient d'être contrôlé par Apple et Google.

Numérique et santé pendant la crise sanitaire

Le 12 juin, une note du ministère de la santé présente une synthèse des projets numériques portés par les pouvoirs publics dans le cadre de la lutte contre l'épidémie. La feuille de route de "l'État plateforme " en matière de santé avait été fixée le 25 avril 2019 autour de trois axes: l'espace numérique de santé destiné aux citoyens, le bouquet de services professionnels et le Health data hub, plateforme de données anonymisées pour la recherche et l'innovation. Pendant l'épidémie, divers outils ont été lancés, soit pour la gestion interne de la crise (suivi de l'épidémie, des lits, des stocks de médicaments...), soit pour aider les citoyens et les professionnels de santé (conseils, contacts), soit pour mettre en valeur des initiatives portées par des entreprises et des chercheurs, enfin, le cadre réglementaire de la télésanté a été assoupli.

\section{$\pi$ Gestion publique}

\section{L'État pendant la crise}

Un sondage Ifop réalisé pour l'Observatoire des politiques publiques dans le cadre d'une étude ActeursPublics/EY (enquête en ligne les 26 et 27 mai auprès de 1003 personnes) indique que $66 \%$ des personnes estiment que les organisations publiques ont bien assuré la continuité des services publics pendant la crise sanitaire. Les hôpitaux récoltent 92 \% de « oui » ou «plutôt oui »; les ministères économiques et financiers $62 \%$. Parmi les enseignements à tirer de la crise sur le fonctionnement des administrations, vient largement en tête la nécessité de « simplifier les procédures administratives pour gagner en productivité ». Selon une note de l'Institut Montaigne qui analyse «l'action publique face à la crise du Covid-19 », la principale faiblesse a été le «piège de la verticalité » de l'administration de l'État qui aurait dû s'appuyer davantage sur la mobilisation et l'adhésion des soignants, des collectivités locales, des entreprises et des citoyens

\section{EUROPE}

\section{Politique européenne}

\section{Pas d'accord sur le plan de relance}

À deux reprises ce mois-ci, lors d'une réunion du Conseil Ecofin le 9 juin et lors d'un Sommet du Conseil européen le 19 juin, les dirigeants européens n'ont pu que constater leur désaccord sur le plan de relance franco-allemand de 750 milliards d'euros. Les quatre «frugaux »/ « radins " (Autriche, Danemark, PaysBas et Suède) sont toujours aussi réservés. Les Vingt-Sept se retrouveront mi-juillet pour tenter de surmonter leurs divergences mais le Premier ministre batave Mark Rutte a déjà prévenu le 19 juin qu'une finalisation de l'accord à cette date était incertaine.

\section{Euro/BCE}

\section{Les bénéficiaires du PEPP}

Le 4 juin 2020, la Banque centrale européenne a publié la répartition des achats de dette qu'elle effectue dans le cadre du programme d'achats d'urgence face à la pandémie (pandemic emergency purchase programme, PEPP). La presse (que ce soit Les Échos ou Le Monde), a constaté que I'Italie en est la principale bénéficiaire. La question d'une extension du programme à hauteur de 600 milliards d'euros fait débat.

\section{Succès du TLTRO 3 auprès des banques}

Les TLTRO (Targeted longer-term refinancing operations), ancienne mesure phare de la BCE pour relancer la très faible dynamique des prix en zone euro, sont proposés aux banques depuis septembre 2014, à raison d'une par trimestre, à la condition expresse que celles-ci accordent davantage de crédits aux ménages et aux entreprises. Le 18 juin 2020, la BCE a annoncé avoir alloué 97,8 Md€ à 143 banques européennes, soit plus du double de ce qui était attendu pour ce troisième prêt de long terme (40 Md€).

\section{त États}

\section{Le plan de relance en Allemagne}

Le 3 juin 2020, le gouvernement de coalition allemand a décidé d'adopter un plan de relance de 130 Md€ comprenant une baisse de la TVA (qui passerait de $19 \%$ à $16 \%$ pour le taux normal, et de $7 \%$ à $5 \%$ pour le taux réduit) et des aides aux familles et à l'investissement, spécialement écologique. 


\section{INTERNATIONAL \\ Les prévisions du FMI}

Lors de la publication de la mise à jour de ses prévisions pour l'économie mondiale, le FMI a estimé que le PIB mondial devrait se contracter de 4,9\% en 2020, c'est-à-dire de 1,9 points de pourcentage de plus que ce qui était prévu dans ses "Perspectives de l'économie mondiale » (PEM) d'avril 2020. La pandémie de COVID-19 aurait ainsi eu un impact négatif plus important que prévu sur l'activité au cours du premier semestre 2020, et la reprise devrait être plus progressive que ce à quoi le FMI s'attendait. En 2021, la croissance mondiale devrait atteindre 5,4\%.

Retrouvez sur www.gestionfinancespubliques. info :

- I'intégralité des REPÈRES d'actualité des finances et de la gestion publiques depuis 2015 -le 15 du mois, les REPÈRES d'actualité du mois précédent

-le 15 janvier 2020, le BEST OF des repères d'actualité de l'année 2019.

- et, en continu, les réflexions des blogueurs de G\&FP.
Aurélien BAUDU (fiscalité et procédure fiscale),

Fabrice BIN (Europe, international), Jean-François CALMETTE (budget de l'État et des opérateurs),

Matthieu HOUSER (finances locales), Michel LE CLAINCHE (comptabilité publique, management public, coordination), Yves TERRASSE (finances sociales). 